that the eigenfrequencies exhibit a tendency toward "locked modes," e.g., an $S$ spin coupled to two $I$ spins will exhibit a single eigenfrequency equal to the root mean square of the individual coupling strengths. For these reasons, and others, ${ }^{1}$ we believe that the present approach has greater promise for the determination of the local geometrical structure of solids.

We are indebted to V. R. Cross for help in the analysis of data.

*Supported in part by the National Science Foundation and in part by the National Institutes of Health.

${ }^{1} \mathrm{~J} . \mathrm{S}$. Waugh, in Proceedings of the Second Specialized Colloque Ampère, Budapest, 1975 (to be published), and to be published.

${ }^{2}$ G. E. Pake, J. Chem. Phys. 16, 327 (1948).

${ }^{3}$ J. H. van Vleck, Phys. Rev. $\overline{74}, 1168$ (1948).

${ }^{4}$ A. Pines, M. G. Gibby, and J. S. Waugh, J. Chem. Phys. 59, 569 (1973).

${ }^{5} \mathrm{U}$. Haeberlen and J. S. Waugh, Phys. Rev. 175, 453 (1968).

${ }^{6}$ W. P. Aue, E. Bartholdi, and R. R. Ernst, J. Chem. Phys. 64, 2229 (1976).

${ }^{7} \mathrm{~J}$. L. Ackerman, J. Tegenfeldt, and J. S. Waugh, J. Am. Chem. Soc. 96, 6843 (1974).

${ }^{8}$ R. K. Hester, J. L. Ackerman, V. R. Cross, and J. S. Waugh, Phys. Rev. Lett. 34,993 (1975); cf. also L. Muller, A. Kumar, T. Baumann, and R. R. Ernst, Phys. Rev. Lett. 32, 1402 (1974).

${ }^{9}$ S. Hartmann and E. L. Hahn, Phys. Rev. 128, 2042 (1962).

\title{
Universal Diamagnetism of Spinless Bose Systems
}

\author{
Barry Simon* \\ Jadwin Physical Laboratory, Princeton University, Princeton, New Jersey 08540 \\ (Received 22 March 1976)
}

I prove that the ground-state energy of an arbitrary system of nonrelativistic spinless Bose particles increases when any (arbitrarily strong or inhomogeneous) magnetic field is turned on.

Let

$$
\begin{aligned}
H(a)=-\sum_{j=1}^{n}\left(2 \mu_{j}\right)^{-1}\left[\nabla_{j}-i e_{j} \overrightarrow{\mathrm{a}}\left(x_{j}\right)\right]^{2} & \\
& +\sum_{j<k} v_{j k}\left(r_{j}-r_{k}\right)+\sum_{j} v_{j}\left(r_{j}\right),
\end{aligned}
$$

where $a, v_{j k}$, and $v_{j}$ are arbitrary real-valued functions. My goal in this note is to prove the following elementary fact that appears to have escaped previous notice:

Theorem.-As an operator on either all squareintegrable $n$-particle functions or on functions with Bose statistics on all of the particles, $H(0)$ has a smaller ground-state energy than $H(a)$.

I emphasize that this result is not coming from a general inequality

$$
(\psi, \boldsymbol{H}(0) \psi) \leqslant(\psi, H(a) \psi)
$$

for all $\psi$, for (2) is false! Equation (2) would imply an inequality on all eigenvalues but it is obvious that in the case $n=1$, and $v$ spherically symmetric, energies of $l \neq 0$ states decrease in lowest-order perturbation theory for a suitable choice of $a$. This remark shows that the theorem fails for fermions for the $v_{i j}$ can be chosen zero! Note also that various analyse ${ }^{1}$ of explicit ex- amples of $H(a)$ have noted the occurrence of an inequality $E(a) \geqslant E(0)$ in these explicit examples.

I will prove the inequality

$$
(|\psi|, H(0)|\psi|) \leqslant(\psi, H(a) \psi) \text {. }
$$

Equation (3) implies the theorem by the variational principle,

$$
\begin{aligned}
E(a) & =\inf _{\|\psi\|=1}(\psi, H(a) \psi) \geqslant \inf (|\psi|, H(0)|\psi|) \\
& \geqslant \inf _{\|\varphi\|=1}(\varphi, H(0) \varphi)=E(0) .
\end{aligned}
$$

Notice that (3), unlike (2), does not imply anything about excited states because $f \rightarrow|f|$ destroys orthogonality. In addition, (3) tells us nothing about the fermion case since $\psi \rightarrow|\psi|$ destroys Fermi statistics.

The inequality (3) follows from a more general inequality $^{2}$ of Kato proven for different purposes. By borrowing only part of his proof one can show (3) easily ${ }^{3}$ : Since $|\psi|^{2}=\psi^{*} \psi$, then with $3 n$-dimensional gradients,

$$
\begin{aligned}
|\psi||\nabla| \psi|| & =\left|\operatorname{Re}\left(\psi^{*} \nabla \psi\right)\right|=\left|\operatorname{Re}\left[\psi^{*}(\nabla-i a) \psi\right]\right| \\
& \leqslant|\psi \|(\nabla-i a) \psi| .
\end{aligned}
$$

Thus $|\nabla| \psi \|^{2}(x) \leqslant|(\nabla-i a) \psi|^{2}(x)$. Integrating over $x$ 
and adding $(\psi, v \psi)=(|\psi|, v|\psi|)$ to both sides yields Eq. (3).

*Alfred P. Sloan Foundation Fellow。 Research partially supported by U。 S。 National Science Foundation Grant No. GP 39048.

${ }^{1}$ See, e.g., K。 Jörgens, Math。Z。 96, 355-372 (1967);
P. R。 dos Santos and H。S。Brandi, Phys。Rev。A (to be published); R. Lavine and $M_{\circ}$ O'Carroll, to be published.

${ }^{2}$ T. Kato, Israel J. Math。13, 135-148 (1973)。Kato proves that $H(0)|\psi| \leqslant \operatorname{Re}[(\operatorname{sgn} \psi) H(a) \psi]$, whence (3) follows by multiplying by $|\psi|$ and integrating.

${ }^{3}$ Since $|\psi|$ is not differentiable, one should really prove (3) as Kato does, letting $\psi_{\epsilon}=\left(|\psi|^{2}+\epsilon^{2}\right)^{1 / 2}$ and then taking $\epsilon$ to zero.

\title{
Saturation of the High-Temperature Normal-State Electrical Resistivity of Superconductors
}

\author{
Z. Fisk* and G. W. Webb† \\ Institute for Pure and Applied Physical Sciences, University of California, San Diego, La Jolla, Califormia 92093
}

(Received 5 January 1976)

\begin{abstract}
The high-temperature, normal-state resistivity of strong-coupled superconducting transition-metal compounds saturates at a value corresponding to an electron mean free path of the order of the interatomic spacing in the compound. This accounts for the nonlinear temperature dependence of the resistivity observed in these compounds at high temperature.
\end{abstract}

The normal-state electrical resistivity $(\rho)$ of a large class of transition-metal superconductors, including the high- $T_{c} A-15$ structure compounds, is known to have a temperature dependence very different from that predicted by the Bloch-Grüneisen theory or Wilson's modification thereof. ${ }^{1-4}$ Near $T_{c}$ the electrical resistance varies approximately as $T^{3}$, consistent with Wilson's theory ${ }^{5}$; this regime joins smoothly to one of strong negative curvature $\left(d^{2} \rho / d T^{2}<0\right)$, the ininflection point, $d^{2} \rho / d T^{2}=0$, being quite generally of order $100^{\circ} \mathrm{K}$; at high temperature the resistivity rises less rapidly than a linear $T$ dependence. We ask how this curvature in the resistivity is related to the occurrence of superconductivity.

One ingredient of the answer is that the electron-phonon coupling $(\lambda)$ in such materials is large. This follows from the magnitude of the observed resistivities: The room-temperature value is high, typically $50-70 \mu \Omega \mathrm{cm}$. In addition, the inflection point of the resistance curve moves to lower temperature as $T_{c}$ increases in closely related sequences of compounds, ${ }^{6}$ consistent with an increased $\lambda$. Anomalous curvature occurs, then, when $\lambda$ is large.

Large $\lambda$ itself, however, does not account for the curvature. Two plausible explanations have been proposed for this curvature. One is the Cohen, Cody, and Halloran model ${ }^{3}$ : A rapid temperature dependence of the density of states at the Fermi level resulting from a nearly empty or full high-density-of-states $d$ band overlying a low-density-of-states $s$ band leads to resistance curves of the kind observed. This model successfully correlates the resistance with a number of other temperature-dependent properties of $\mathrm{Nb}_{3} \mathrm{Sn}, \mathrm{V}_{3} \mathrm{Si}$, and $\mathrm{V}_{3} \mathrm{Ga}$. The other explanation is that of Allen et al. ${ }^{7}$ : Hardening of lattice vibrational modes as $T$ increases results in a resistivity which rises with less than a linear $T$ dependence. On the basis of neutron data for $\mathrm{V}_{3} \mathrm{Si}$, they can account for about half the curvature observed in the resistivity of $\mathrm{V}_{3} \mathrm{Si}$ from $T_{c}$ to room temperature.

We belive that, while the above treatments are appropriate to these materials, at high temperatures a different effect dominates. Figure 1 shows data on the electrical resistivity of $A-15$ structure $\mathrm{Nb}_{3} \mathrm{Sn}\left(T_{c}=18^{\circ} \mathrm{K}\right)$ and single crystal $\mathrm{Nb}_{3} \mathrm{Sb}\left(T_{c}=0.2^{\circ} \mathrm{K}\right)$. Data for $\mathrm{Nb}_{3} \mathrm{Sn}$ were read from a graph in Ref. 1. Knapp, Bader, and Fisk have measured the heat capacity of both these compounds between 2 and $400^{\circ} \mathrm{K}^{8}$ The electronic specific heat $\gamma$ for $\mathrm{Nb}_{3} \mathrm{Sb}$ is $1.1 \mathrm{~mJ} /{ }^{\circ} \mathrm{K}^{2}$ gramatom, more than a factor of 10 less than that of the isostructural $\mathrm{Nb}_{3} \mathrm{Sn}$. In addition, analysis of the anharmonic contribution to the heat capacity shows that the $\mathrm{Nb}_{3} \mathrm{Sb}$ lattice softens as $T$ inc reases, opposite to $\mathrm{Nb}_{3} \mathrm{Sn}$. It is unlikely, therefore, that the previous two explanations apply to $\mathrm{Nb}_{3} \mathrm{Sb}$, yet its resistivity is very similar to that of $\mathrm{Nb}_{3} \mathrm{Sn}$ 